\title{
Early childhood infections, antistreptococcal and basal ganglia antibodies in adult ADHD: a preliminary study
}

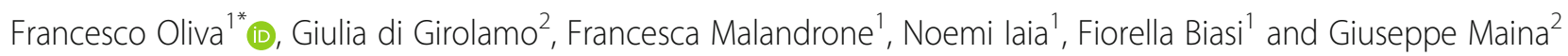

\begin{abstract}
Background: To explore the relationship between adult Attention Deficit/ Hyperactivity Disorder (ADHD), antistreptococcal titers, ABGA, and recurrent infections during early childhood.

Method: Childhood history of recurrent infections and a blood sample were collected in a sample of DSM-IV adult outpatients with ADHD. The anti-streptolysin O (ASO), anti-deoxyribonuclease B (anti-DNase B), and anti-basal ganglia antibodies (ABGA) titers were determined in patient plasma by enzyme-linked immunosorbent assay (ELIS A). Titers positivity was evaluated following manufacturer's specifications. Absolute titers were also collected as continuous variables.

Results: Fourteen out of 22 (63.6\%) have had recurrent infections in childhood (i.e., seven, 31.8\%, have had tonsillitis or adenoiditis and seven, 31.8\%, have had any other infections). Eighteen patients (81.9\%) were positive for anti-DNase B, five (22.7\%) for ASO, and 4 (18.2\%) were positive for both of them. Five participants (22.7\%) were ABGA positive, whereas only two (9.1\%) were positive for all three antibodies.

Conclusions: patients with ADHD might be more prone to infections during childhood and subclinical streptococcal infections during adulthood. Moreover, they seem to have an increased risk for basal ganglia autoimmunity in adulthood. Both infections and the ensuing acquired autoimmunity could influence the neurodevelopmental process, by contributing, at least in part, to the ADHD pathogenesis.
\end{abstract}

Keywords: ADHD, Group a streptococcus, Anti-streptolysin O, Anti-deoxyribonuclease B, ABGA, Basal ganglia, Adult

\section{Background}

ADHD is one of the most prevalent neurodevelopmental disorders and it is characterized by a persistent pattern of inattention and/or hyperactivity and/or impulsivity, leading to functional impairment [1] and several lifetime consequences [2]. Despite ADHD arises in childhood, it can persist in adulthood in about half of patients as a

\footnotetext{
* Correspondence: francesco.oliva@unito.it

'Department of Clinical and Biological Science, University of Turin, Orbassano (TO), Italy

Full list of author information is available at the end of the article
}

full-criteria disorder $(45-57 \%)[3,4]$, whereas a further $37 \%$ can show impairing symptoms or a subthreshold disorder at the 10 years after follow-up [3]. Neuroimaging and neurophysiological studies have consistently documented that improvement with age in core symptoms are accompanied by functional changes in cortical (right inferior frontal and inferior parietal/precuneus) and cerebellar regions [5, 6], whereas basal ganglia anomalies (i.e., right caudate) seem to be a peculiar lifetime feature of ADHD, regardless of adult remission [6, 7]. Studies on structural anomalies and age-by-diagnosis

(C) The Author(s). 2020 Open Access This article is licensed under a Creative Commons Attribution 4.0 International License, which permits use, sharing, adaptation, distribution and reproduction in any medium or format, as long as you give appropriate credit to the original author(s) and the source, provide a link to the Creative Commons licence, and indicate if changes were made. The images or other third party material in this article are included in the article's Creative Commons licence, unless indicated otherwise in a credit line to the material. If material is not included in the article's Creative Commons licence and your intended use is not permitted by statutory regulation or exceeds the permitted use, you will need to obtain permission directly from the copyright holder. To view a copy of this licence, visit http://creativecommons.org/licenses/by/4.0/ The Creative Commons Public Domain Dedication waiver (http://creativecommons.org/publicdomain/zero/1.0/) applies to the data made available in this article, unless otherwise stated in a credit line to the data. 
interaction in the caudate and putamen have also supported the pivotal role of subcortical basal ganglia anomalies in the pathophysiology of ADHD [8, 9].

Although the etiopathogenesis of ADHD is still unclear, a gene by environment model has been proposed to explain the high family history of the disorder $[2,10$, 11]. Probably, a variable and complex interaction between predisposing genetic and environmental factors during the early months or years of life could affect the normal path of neurodevelopment. Considering the above-mentioned structural and functional evidences, environmental factors influencing basal ganglia development should be worthy of investigation. For instance, a specific vascular vulnerability of the striatum due to its positioning in a border zone of arterial supply has already been suggested by a case-control study reporting on hypoxic and other perinatal complications [12] and by a review of some in vivo studies [13]. A more recent study has documented the negative impact of exposure to polycyclic aromatic hydrocarbons on the caudate nucleus, even below the legislated annual target levels established in the European Union [14].

Over the last 20 years, the recognition of the role of some infections (e.g., group A streptococcus - GAS) in determining acute, somewhat mixed, movement (i.e., Sydenham's chorea) and psychiatric disorders (i.e., obsessivecompulsive disorders, tic disorder, ADHD), have increased the attention to infective agents as possible causes of childhood neurodevelopmental disorders, involving basal ganglia [15-18]. A molecular mimicry mechanism has also been proposed to explain the enduring symptoms subsequent to GAS infection remission [19, 20]. Moreover, high titers of GAS infection markers, i.e., anti-streptolysin $\mathrm{O}$ (ASO) and anti-deoxyribonuclease B (anti-DNase B), and anti-basal ganglia antibodies (ABGA) were found in this type of disorders [21-23].

Since Kiessling and colleagues [22] have reported a higher prevalence of distractibility and hyperactivity among patients with post-streptococcal movement disorders than in controls, a growing number of subsequent studies have specifically investigated, in different ways, the relationship between GAS infections, basal ganglia autoimmunity and ADHD with conflicting results [24-28].

ADHD appears to play an important role in poststreptococcal basal ganglia disorder at least as much as obsessive-compulsive and tic disorders even when considered alone: higher titers of GAS infection markers were found in children with ADHD than in those with obsessive-compulsive or tic disorder; ADHD and even more hyperactivity symptoms significantly predicted anti-DNase B titer; and ASO titers were correlated with basal ganglia volume in children with ADHD [26].

More recently, three case-control studies have focused on GAS infection and ABGA titers of children with
ADHD and without comorbid obsessive-compulsive/tic disorders [24, 27, 28]. Only one study found higher ABGA titers among children with ADHD than controls (30\% vs. $15 \%,[28]$ ), whereas higher titers of ASO and anti-DNase B were reported in children with ADHD by two out of three studies [27, 28]. All those inquiring about GAS infection collecting pharyngeal swabs reported higher rates of positive culture among children with ADHD than controls [24, 27]. Regardless of the specific strengths and limitations, they all were conducted on child populations and, taken together with above-mentioned studies, they suggest a possible predisposition of patients with ADHD to be infected by GAS, involving basal ganglia development.

To the best of our knowledge, no studies have assessed GAS antibodies and ABGA titers in adult patients with ADHD in an attempt to confirm the lifespan vulnerability of this population to GAS infection, which could also, in some cases, involve basal ganglia autoimmunity.

The aim of the present preliminary study was to explore ASO, anti-DNase B, and ABGA titers in a sample of adult outpatients with ADHD.

\section{Methods}

This preliminary study was conducted at the adult ADHD outpatient center of the San Luigi Gonzaga University Hospital (Orbassano, Turin, Italy), between July 1, 2019 and September 1, 2019, on a consecutive sample of patients newly diagnosed with adult ADHD according to DSM-IV-TR criteria (age $>18$ years). According to the outpatient service routine, all patients had been screened for adult ADHD using the Adult ADHD Self Reporting Scale (ASRS-1.1) screener, and then the DSM-IV ADHD diagnosis was confirmed through the Diagnostic Interview for Adult ADHD (DIVA 2.0). The severity of ADHD symptoms was rated by Adult ADHD Investigation Rating Scale (AISRS).

All patients were asked to participate in the study signing a written informed consent and a unique identification code was assigned to each patient, in order to maintain data anonymity and patient confidentiality. The research protocol of the present observational study was approved by the Research Ethics Committee of the San Luigi Gonzaga University Hospital (Orbassano, Turin, Italy), therefore the study was conducted in accordance with the Helsinki Declaration.

During the assessment process, information about the history of both infections and psychiatric disorders were collected and patients were also assessed for other psychiatric disorders through an in-depth psychiatric interview. Recurrent infections in childhood (i.e., under 12) were defined as at least three symptomatic episodes of infection with fever (e.g., tonsillitis, adenoiditis, glomerulonephritis, or others) per year for at least 1 year. 
Blood samples from patients were collected in potassium ethylenediaminetetraacetic acid (EDTA) - containing tubes (BD Vacutainer ${ }^{\circ}$ spray-coated K2EDTA, Becton, Dickinson and Company, Franklin Lakes, NJ, USA) at the end of each recruitment. Plasma was separated by centrifugation at $24,104 \mathrm{x}$ g at $4{ }^{\circ} \mathrm{C}$ for $10 \mathrm{~min}$ and immediately stored at $-80^{\circ} \mathrm{C}$ ready for analyses.

Commercial, ready-to-use, microwell ELISA kits were used for the quantitative determination of plasma concentration of ASO (Cat. No MBS038268, MyBioSource Inc., San Diego, USA), anti-DNase B (Cat. No MBS7226468, MyBioSource Inc., San Diego, USA), and ABGA (Cat. No MBS706650, MyBioSource Inc., San Diego, USA). Sample absorbance values were detected in a 96-multiwell plate reader (Model 680 Microplate Reader, Bio-Rad, Milan, Italy) using dual-wavelength recording modes at $450 \mathrm{~nm}$ and $655 \mathrm{~nm}$ (the latter used as reference). ELISA protocols instructions, and threshold values for adults provided by the manufacturer were adopted. . Thereby, the following values defined patient positivity: $\mathrm{ASO}>200 \mathrm{U} / \mathrm{ml}$; anti-DNase $\mathrm{B}>86 \mathrm{ng} / \mathrm{ml}$; ABGA $>1.18$ (as the ratio referred to the negative control optical density) but absolute titers values were also collected as continuous variables.

\section{Clinical assessment tools}

The ASRS 1.1 [29] is a six-item self-report screener for adult ADHD, based on the DSM-IV-TR criteria. It was developed by the World Health Organization (WHO) and then validated internationally [30]. The screening is considered positive when at least four answers are above cut-off value.

The DIVA 2.0 [31] is a validated [32] semistructured interview to assess the adult patients, according to the DSM-IV criteria. It investigates DSM-IV inattentive and hyperactivity/impulsivity symptoms (criterion A) in both childhood and adulthood, using several real-life examples to support each question inquiring about 18 DSMIV criteria. It also includes explicit questions for age at onset (i.e., the presence of symptoms before the age of 7 years, criterion B) and other primary psychiatric and/or substance use disorders (which could better explain the reported symptoms, criterion E). The last section consists in an in-depth evaluation of the main areas of functioning impacted by ADHD symptoms (criteria $\mathrm{C}$ and D). Furthermore, the collateral information should be reported in both content and source (i.e., the presence of parents/third parties; school reports).

The AISRS is a validated [33] 18-item scale matching the DSM-IV criteria. It is divided into two subscales of nine items each, investigating inattentive and hyperactivity/impulsivity, respectively. The items are provided with examples to minimize interrater variability. The scoring system ranges from zero (none) to three (severe). The maximum score for each subscale is 27 points, with a maximum total score of 54 .

\section{Statistical analysis}

All analyses and calculations were performed using RStudio for MAC OS (Version 1.1.383, RStudio Inc., Boston, MA).

The positivity rate for every titer was calculated. The distributions of continuous variables were tested by Shapiro-Wilk's test. A $p$-value of 0.05 was used to designate statistical significance. Mean and standard deviation (SD) were used to report normally distributed variables, whereas median (Mdn) and interquartile range (IQR) were used for nonnormally distributed ones.

\section{Results}

A sample of 22 adult outpatients with ADHD was recruited. The sociodemographic and clinical features of the sample are summarized in Table 1. Little more than half of the patients were male. The age was not normally distributed (Shapiro-Wilk's test $\mathrm{W}=0.868, p<.001$ ). Most of the patients have reached a middle or high school diploma, but more than a fifth of them have got a degree. Although the majority of the sample referred from GPs, psychiatrists, or child neuropsychiatrists, less than half accessed the adult ADHD center on their own (Table 1). .

Little more than half of the sample had a predominantly inattentive subtype ADHD. The rate of psychiatric comorbidity was high but no patient has ever suffered from tic disorder, obsessive-compulsive disorder or Sydenham's chorea (Table 1).

Little more than a third of the sample has had recurrent infections with fever in childhood (Table 1)).

As regards antistreptococcal antibodies, 18 patients (81.9\%) had positive anti-DNase B titer, five (22.7\%) had positive ASO titer, four (18.2\%) had them both positive. Five (22.7\%) patients were ABGA positive, whereas only two $(9.1 \%)$ resulted positive for all three titers. All titers were nonnormally distributed in the examined population (ASO, Median $=114.5$, IQR $=82.2$, Shapiro-Wilk's test $\mathrm{W}=.792, p<.001$; anti-DNase $\mathrm{B}, \quad$ Median $=95$, $\mathrm{IQR}=17$, Shapiro-Wilk's test $\mathrm{W}=.915, p=.049$; ASO, Median $=.54$, IQR $=.17$, Shapiro-Wilks W test $=.661$, $p<.001)$. Within sample subgroups comparison could not be performed due to low numerosity.

\section{Discussion}

This is the first report on anti-GAS antibodies and ABGA titers in adult patients with ADHD. Although it is a preliminary study, the magnitude of GAS infections and basal ganglia autoimmunity in adult patients with ADHD cannot be considered negligible as a large proportion of the sample was anti-DNase B positive (index 
Table 1 Sociodemographic and clinical features of the sample $(N=22)$

\begin{tabular}{ll}
\hline Features & Value \\
\hline Male, $\mathrm{n}(\%)$ & $12(54.5 \%)$ \\
Age (years), Mdn \pm IQR & $30 \pm 12$ \\
Education, $\mathrm{n}(\%)$ & \\
Middle school diploma & $9(40.9)$ \\
High school diploma & $8(36.4)$ \\
Bachelor's degree & $2(9.1)$ \\
Master's degree & $3(13.6)$ \\
Occupational status, $\mathrm{n}(\%)$ & \\
Employed & $11(50)$ \\
Unemployed & $6(27.3)$ \\
Students & $5(22.7)$ \\
Reference to ADHD outpatient center, $\mathrm{n}(\%)$ & \\
Autonomous & $10(45.5)$ \\
General Practitioner & $4(18.2)$ \\
Child neuropsychiatrist & $2(9.1)$ \\
ADHD subtype, $\mathrm{n}$ (\%) & \\
Predominantly inattentive & $12(54.5)$ \\
Predominantly hyperactive/impulsive & $1(4.5)$ \\
Combined & $1(4.5)$ \\
Positive family history of psychiatric disorders, $\mathrm{n}(\%)$ & $10(45.5)$ \\
Positive family history of ADHD, $\mathrm{n}(\%)$ & $8(36.4)$ \\
Recurrent infections during childhood, $\mathrm{n}(\%)$ & $6(27.3)$ \\
Tonsillitis or adenoiditis & $8(36.4)$ \\
with Surgical removal & $14(63.6 \%)$ \\
\hline
\end{tabular}

of prior GAS infections), more than a quarter of the sample still showed ASO titer positivity (index of recent GAS infection) and the same amount presents ABGA positivity (index of acquired autoimmunity). The comparison with previous studies conducted on children with ADHD is challenging because of methods heterogeneity, albeit they all excluded patients with tics, obsessive-compulsive disorder, and Sydenham's chorea. On average, the positivity rate of ASO titers reported by previous studies (ranging from 50 to $60 \%,[24,28]$ ) doubled that detected in the present preliminary investigation, even considering the well-recognized age-related decrease of the threshold value [34]. Conversely, the positivity rate of anti-DNase B in our adult sample was higher than that reported by a prior study on children with ADHD (81.3\% vs. 60\%, [24]). The elevation of both ASO and anti-DNase B above the threshold was found in less than half proportion of patients than that reported by two of the prior studies (ranging from 50 to $60 \%,[24,27])$.

All patients were recruited and tested for titers during summer, the season having the fewest GAS outbursts [34]. On the one hand, this can partially explain the lower proportion of positive ASO titers (index of recent infection) than that registered in previous studies enrolling child patients during all seasons $[24,27,28]$. On the other hand, it provides further support to our findings because they should be considered an underestimation of the actual scenario. Retrospectively collected data on childhood history of infection suggest a distinctive immune vulnerability of ADHD as almost two-thirds of the sample showed a variety of recurrent infections, half of which were tonsillitis or adenoiditis. To our knowledge, this is the first report on the history of recurrent infection in adult ADHD. Only a previous study collected this data in children with ADHD, finding that $18 \%(n=7)$ of the sample had a history of GAS infections [24]. All participants of the present preliminary study were nonremitter adult ADHD, thus adults who have had ADHD in childhood but not having current ADHD were not enrolled (i.e., remitters and subthreshold ADHD patients). Bearing in mind the ADHD remission rate from childhood to adulthood $(45-57 \%,[3,4])$, we can suppose that a history of infections and thus a possible vulnerability to them might be a common feature of persistent adult ADHD. Future studies comparing remitters and non-remitter adults should be conducted to confirm this suggestion. Furthermore, the poor reliability of retrospective data collection could partially explain this considerable discrepancy in rate history of infections between adults and children and can not allow to discern streptococcus-related from other infections.

Even more emerged about basal ganglia autoimmunity as over a fifth of the sample had positive ABGA titers, consistently with a previous report regarding Italian children with ADHD (25\%, [28]). This rate was also in line with that found in adults affected by obsessivecompulsive disorder (19.8\%, [35]), which represents one of the most known neuropsychiatric manifestation of post-streptococcal autoimmune basal ganglia disorders [36]. ADHD seems to be even more prevalent than obsessive-compulsive disorder in adult populations $(2.8 \%$ vs. $1.2 \%,[4,37])$ and, as mentioned above, the implication of structural and functional anomalies of basal ganglia in its pathophysiology has been well-recognized by neuroimaging studies $[5,6,8,9]$. However, the relationship between ADHD and post-infective basal ganglia disorders is little investigated, especially during adulthood, when the neurodevelopment is expected to be complete, 
thus neither infections nor the autoimmunity process can longer affect its course.

The main aim of the present study was to provide preliminary findings on the usefulness of ASO, anti-DNase $B$, and $A B G A$ titers measurement in adult patients with ADHD. In this respect, we used easily available, standard, ready-to-use ELISA kit tests for the quantitative determination of blood titers that increased the reproducibility of our research and findings.

Nevertheless, different limitations should be taken into account. First of all, the sample size of the present study was small, although it was not so far from those of some clinical groups of previous case-control studies conducted on children ( $n=20$, [28] and $n=22$, [27]), which however included a more or less appropriate control group (i.e., healthy children or patients with neurological disorders). In this regard, a noteworthy concern for future studies could be establishing the right eligibility criteria for a control group, given the impressive high psychiatric comorbidity rate of ADHD in adulthood (i.e., non-ADHD patients matched for comorbidity, enrollment season and age?). Another limitation can be recognized in current GAS infection detection because the inclusion of a rapid strep test or a pharyngeal swab culture would have been highly recommended to reach a more informative assessment.

Further studies including appropriate controls should be conducted on wider samples to confirm our preliminary findings, providing more solid evidence to support the already suggested vulnerability of patients with ADHD to some type of infections in childhood and perhaps also in adulthood [26, 28]. Nevertheless, to evaluate the trajectories of the relationship between childhood infections and ADHD development, further studies might consider ADHD remitters (i.e., adults without current ADHD but who have had ADHD in childhood). Notably, future studies may focus on a potential genetic predisposition to infection or other environmental factors affecting the neurodevelopment of the basal ganglia.

\section{Conclusion}

According to the present preliminary study, patients with ADHD might be prone to infections during childhood, subclinical streptococcal infections in adulthood, and they seem to have a high risk for basal ganglia autoimmunity as adults. Both infections and the ensuing acquired autoimmunity may influence the neurodevelopmental process, by contributing, at least in part, to the ADHD pathogenesis. ASO, anti-DNase B and ABGA titers seem to be easy to determine by using specific ELISA kits. It may be possible that a childhood predisposition to infection, which persists into adulthood as antistreptococcal titer positivity, might also contribute to the pathogenesis of ADHD and even to its persistence.
Future controlled studies should confirm on wider samples our findings on streptococcal infections, autoimmunity, and ADHD. As for some other neuropsychiatric disorders, ADHD may be related to streptococcal infections or basal ganglia autoimmunity and this could have implications on pharmacological treatment.

\begin{abstract}
Abbreviations
ADHD: Attention Deficit/ Hyperactivity Disorder; ASO: Anti-streptolysin O; anti-DNase B: Anti-deoxyribonuclease B; ABGA: Anti-basal ganglia antibodies; EDTA: Ethylenediaminetetraacetic acid; ASRS-1.1: Adult ADHD Self Reporting Scale; DIVA: Diagnostic Interview for Adult ADHD; AISRS: Adult ADHD

Investigation Rating Scale; ELISA: Enzyme-linked immunosorbent assay
\end{abstract}

\section{Acknowledgements}

Researchers would like to thank all patients involved in the study for their valuable contribution.

\section{Authors' contributions}

FO and Gd conceived the study and drafted the manuscript. Gd collected blood samples and managed the recruitment process. NI and FB conducted laboratory analyses. FM collected and analyzed data. GM coordinated times and stages of the study. All authors read and approved the final manuscript.

\section{Funding}

This work was supported by the Clinical and Biological Sciences Department of the University of Turin (Local research funding call, B quota, No

OLIF_RILO_17_01). The funding body did not play any role in the design of the study and collection, analysis, and interpretation of data, and in writing the manuscript.

\section{Availability of data and materials \\ The data that support the findings of this study are available on request from the corresponding author. The data are not publicly available due to privacy or ethical restrictions.}

\section{Ethics approval and consent to participate}

The research protocol of the present observational study was approved by the Research Ethics Committee of the San Luigi Gonzaga University Hospital (Orbassano, Turin, Italy), therefore the study was conducted in accordance with the Helsinki Declaration.

\section{Consent for publication}

Not applicable.

\section{Competing interests}

The authors declare no potential conflicts of interest with respect to the research, authorship, or publication of this article.

\section{Author details}

${ }^{1}$ Department of Clinical and Biological Science, University of Turin, Orbassano (TO), Italy. "Department of Neurosciences "Rita Levi Montalcini", University of Turin, Turin, Italy.

Received: 16 April 2020 Accepted: 7 November 2020

Published online: 18 November 2020

References

1. American Psychiatric Association. Diagnostic and statistical manual of mental disorders, 5th Edition: DSM-5. Arlington: American Psychiatric Publishing; 2013.

2. Kooij JJS, Bijlenga D, Salerno L, Jaeschke R, Bitter I, Balázs J, et al. Updated European Consensus Statement on diagnosis and treatment of adult ADHD. European Psychiatry. 2019;56:14-34.

3. Biederman J, Petty CR, Evans M, Small J, Faraone SV. How persistent is ADHD? A controlled 10-year follow-up study of boys with ADHD. Psychiatry Res. 2010;177:299-304. 
4. Fayyad J, Sampson NA, Hwang I, Adamowski T, Aguilar-Gaxiola S, AlHamzawi A, et al. The descriptive epidemiology of DSM-IV Adult ADHD in the World Health Organization World Mental Health Surveys. Atten Defic Hyperact Disord. 2017;9:47-65.

5. Halperin JM, Schulz KP. Revisiting the role of the prefrontal cortex in the pathophysiology of attention-deficit/hyperactivity disorder. Psychol Bull. 2006;132:560-81.

6. Szekely E, Sudre GP, Sharp W, Leibenluft E, Shaw P. Defining the Neural Substrate of the Adult Outcome of Childhood ADHD: A Multimodal Neuroimaging Study of Response Inhibition. Am J Psychiatry. 2017;174:86776.

7. Dang LC, Samanez-Larkin GR, Young JS, Cowan RL, Kessler RM, Zald DH. Caudate asymmetry is related to attentional impulsivity and an objective measure of ADHD-like attentional problems in healthy adults. Brain Struct Funct. 2016:221:277-86.

8. Greven CU, Bralten J, Mennes M, O'Dwyer L, van HKJE, Rommelse N, et al. Developmentally Stable Whole-Brain Volume Reductions and Developmentally Sensitive Caudate and Putamen Volume Alterations in Those With Attention-Deficit/Hyperactivity Disorder and Their Unaffected Siblings. JAMA Psychiatry. 2015;72:490-9.

9. Seidman LJ, Valera EM, Makris N. Structural Brain Imaging of AttentionDeficit/Hyperactivity Disorder. Biological Psychiatry. 2005;57:1263-72.

10. Faraone SV, Perlis RH, Doyle AE, Smoller JW, Goralnick JJ, Holmgren MA, et al. Molecular Genetics of Attention-Deficit/Hyperactivity Disorder. Biological Psychiatry. 2005;57:1313-23.

11. Faraone SV, Asherson P, Banaschewski T, Biederman J, Buitelaar JK, RamosQuiroga JA, et al. Attention-deficit/hyperactivity disorder. Nat Rev Dis Primers. 2015:1:15020.

12. Sprich-Buckminster S, Biederman J, Milberger S, Faraone SV, Lehman BK. Are perinatal complications relevant to the manifestation of ADD? Issues of comorbidity and familiality. J Am Acad Child Adolesc Psychiatry. 1993;32: 1032-7

13. Calabresi P, Centonze D, Gubellini P, Marfia GA, Pisani A, Sancesario G, et al. Synaptic transmission in the striatum: from plasticity to neurodegeneration. Progress Neurobiol. 2000;61:231-65.

14. Mortamais M, Pujol J, van Drooge BL, Macià D, Martínez-Vilavella G, Reynes $C$, et al. Effect of exposure to polycyclic aromatic hydrocarbons on basal ganglia and attention-deficit hyperactivity disorder symptoms in primary school children. Environ Int. 2017;105:12-9.

15. Dale RC, Heyman I. Surtees R a. H, Church AJ, Giovannoni G, Goodman R, et al. Dyskinesias and associated psychiatric disorders following streptococcal infections. Arch Dis Child. 2004;89:604-10.

16. Dale RC, Brilot F. Autoimmune Basal Ganglia Disorders. J Child Neurol. 2012; 27:1470-81.

17. Mercadante MT, Busatto GF, Lombroso PJ, Prado L, Rosário-Campos MC, do Valle $R$, et al. The psychiatric symptoms of rheumatic fever. Am J Psychiatry. 2000;157:2036-8.

18. Swedo SE, Leonard HL, Garvey M, Mittleman B, Allen AJ, Perlmutter S, et al. Pediatric autoimmune neuropsychiatric disorders associated with streptococcal infections: clinical description of the first 50 cases. Am J Psychiatry. 1998;155:264-71.

19. Cunningham MW. Molecular Mimicry, Autoimmunity and Infection: The Crossreactive Antigens of Group A Streptococci and their Sequelae. Microbiol Spectr. 2019;7. https://doi.org/10.1128/microbiolspec.GPP3-0045-2018.

20. Kirvan CA, Swedo SE, Kurahara D, Cunningham MW. Streptococcal mimicry and antibody-mediated cell signaling in the pathogenesis of Sydenham's chorea. Autoimmunity. 2006;39:21-9.

21. Church AJ, Cardoso F, Dale RC, Lees AJ, Thompson EJ, Giovannoni G. Antibasal ganglia antibodies in acute and persistent Sydenham's chorea. Neurology. 2002;59:227-31.

22. Kiessling LS, Marcotte AC, Culpepper L. Antineuronal antibodies in movement disorders. Pediatrics. 1993:92:39-43.

23. Martino D, Church A, Giovannoni G. Are antibasal ganglia antibodies important, and clinically useful? Practical Neurology. 2007;7:32-41.

24. Aguilera-Albesa S, Crespo-Eguílaz N, Del Pozo JL, Villoslada P, Sánchez Carpintero R. Anti-Basal Ganglia Antibodies and Streptococcal Infection in ADHD. J Atten Disord. 2018;22:864-71.

25. Kiessling LS, Marcotte AC, Culpepper L. Antineuronal antibodies: tics and obsessive-compulsive symptoms. J Dev Behav Pediatr. 1994;15:421-5.

26. Peterson BS, Leckman JF, Tucker D, Scahill L, Staib L, Zhang H, et al. Preliminary findings of antistreptococcal antibody titers and basal ganglia volumes in tic, obsessive-compulsive, and attention deficit/hyperactivity disorders. Arch Gen Psychiatry. 2000:57:364-72.

27. Sanchez-Carpintero R, Albesa SA, Crespo N, Villoslada P, Narbona J. A preliminary study of the frequency of anti-basal ganglia antibodies and streptococcal infection in attention deficit/hyperactivity disorder. J Neurol. 2009;256:1103-8.

28. Toto M, Margari F, Simone M, Craig F, Petruzzelli MG, Tafuri S, et al. Antibasal Ganglia Antibodies and Antistreptolysin O in Noncomorbid ADHD. J Atten Disord. 2015:19:965-70.

29. Kessler RC, Adler L, Ames M, Demler O, Faraone S, Hiripi E, et al. The World Health Organization Adult ADHD Self-Report Scale (ASRS): a short screening scale for use in the general population. Psychol Med. 2005;35:245-56.

30. Adler LA, Spencer T, Faraone SV, Kessler RC, Howes MJ, Biederman J, et al, Validity of Pilot Adult ADHD Self-Report Scale (ASRS) to Rate Adult ADHD Symptoms. Annals of Clinical Psychiatry. 2006:18:145-8.

31. Kooij JJS, Adult ADHD. Diagnostic Assessment and Treatment. 3rd ed. London: Springer-Verlag; 2013. https://doi.org/10.1007/978-1-4471-4138-9.

32. Ramos-Quiroga JA, Nasillo V, Richarte V, Corrales M, Palma F, Ibáñez P, et al. Criteria and Concurrent Validity of DIVA 2.0: A Semi-Structured Diagnostic Interview for Adult ADHD. J Atten Disord. 2019:23:1126-35.

33. Spencer TJ, Adler LA, null MQ, Saylor KE, Brown TE, Holdnack JA, et al. Validation of the adult ADHD investigator symptom rating scale (AISRS). J Atten Disord. 2010;14:57-68.

34. Shulman ST, Tanz RR, Kabat W, Kabat K, Cederlund E, Patel D, et al. Group A streptococcal pharyngitis serotype surveillance in North America, 2000-2002. Clin Infect Dis. 2004;39:325-32.

35. Nicholson TRJ, Ferdinando S, Krishnaiah RB, Anhoury S, Lennox BR, MataixCols $\mathrm{D}$, et al. Prevalence of anti-basal ganglia antibodies in adult obsessivecompulsive disorder: cross-sectional study. Br J Psychiatry. 2012;200:381-6.

36. Baj J, Sitarz E, Forma A, Wróblewska K, Karakuła-Juchnowicz H. Alterations in the Nervous System and Gut Microbiota after $\beta$-Hemolytic Streptococcus Group A Infection-Characteristics and Diagnostic Criteria of PANDAS Recognition. Int J Mol Sci. 2020;21. https://doi.org/10.3390/ijms21041476.

37. Ruscio AM, Stein DJ, Chiu WT, Kessler RC. The Epidemiology of ObsessiveCompulsive Disorder in the National Comorbidity Survey Replication. Mol Psychiatry. 2010;15:53-63.

\section{Publisher's Note}

Springer Nature remains neutral with regard to jurisdictional claims in published maps and institutional affiliations.
Ready to submit your research? Choose BMC and benefit from:

- fast, convenient online submission

- thorough peer review by experienced researchers in your field

- rapid publication on acceptance

- support for research data, including large and complex data types

- gold Open Access which fosters wider collaboration and increased citations

- maximum visibility for your research: over $100 \mathrm{M}$ website views per year

At BMC, research is always in progress.

Learn more biomedcentral.com/submissions 